\title{
Philosophiques
}

\section{Bolzano sur la structure des propositions et le rôle sémantique des propriétés}

\section{Benjamin Schnieder}

Volume 30, numéro 1, printemps 2003

Bernard Bolzano. Philosophie de la logique et théorie de la connaissance

URI : https://id.erudit.org/iderudit/007733ar

DOI : https://doi.org/10.7202/007733ar

Aller au sommaire du numéro

Éditeur(s)

Société de philosophie du Québec

ISSN

0316-2923 (imprimé)

1492-1391 (numérique)

Découvrir la revue

Citer cet article

Schnieder, B. (2003). Bolzano sur la structure des propositions et le rôle sémantique des propriétés. Philosophiques, 30(1), 83-103.

https://doi.org/10.7202/007733ar
Résumé de l'article

Bernard Bolzano développe une théorie exhaustive et très élaborée des propositions comme entités structurées et composées de concepts. L'une de ses thèses principales consiste à dire que toutes les propositions ont en commun la même structure : « $A-\mathrm{a}$ - (la propriété) $b$ ». Cet article examine le rôle que jouent les propriétés eu égard à cette thèse. Lorsque les propriétés figurent dans les théories sémantiques standards, elles sont généralement conçues comme des entités partageables, en d'autres mots, comme des universaux. Je montre que (contrairement à ce qui fait consensus dans la littérature) Bolzano croyait que ce sont bien plutôt des propriétés particularisées qui tombent sous la représentation-prédicat d'une proposition. De là émerge une sémantique plutôt inhabituelle : une proposition de la forme [A-a - (la propriété) b] est vraie ssi une des propriétés particularisées qui se tiennent sous la représentation-prédicat $[\mathrm{b}]$ inhère au sujet de la proposition, c'est-à-dire l'entité dénotée par la représentation-sujet [A]. 


\title{
Bolzano sur la structure des propositions et le rôle sémantique des propriétés
}

\author{
BENJAMIN SCHNIEDER \\ Université de Hambourg \\ b.schnieder@gmx.de
}

\begin{abstract}
RÉSUMÉ. - Bernard Bolzano développe une théorie exhaustive et très élaborée des propositions comme entités structurées et composées de concepts. L'une de ses thèses principales consiste à dire que toutes les propositions ont en commun la même structure : «A-a - (la propriété) $b$ ». Cet article examine le rôle que jouent les propriétés eu égard à cette thèse. Lorsque les propriétés figurent dans les théories sémantiques standards, elles sont généralement conçues comme des entités partageables, en d'autres mots, comme des universaux. Je montre que (contrairement à ce qui fait consensus dans la littérature) Bolzano croyait que ce sont bien plutôt des propriétés particularisées qui tombent sous la représentation-prédicat d'une proposition. De là émerge une sémantique plutôt inhabituelle : une proposition de la forme [A - a - (la propriété) b] est vraie ssi une des propriétés particularisées qui se tiennent sous la représentation-prédicat [b] inhère au sujet de la proposition, c'est-à-dire l'entité dénotée par la représentation-sujet $[A]$.
\end{abstract}

ABSTRACT. - Bernard Bolzano developed a highly elaborate and comprising account of propositions as structured entities composed of concepts. One of his main contentions was that all propositions share a common structure : " $A$ - has - (the property) $b$ ". The main part of my paper is a discussion of the role which properties play for this thesis and thus in Bolzano's semantics. Where properties feature as semantic values in standard semantic theories they are (at least in general) conceived of as shareable entities, in other words, as universals. I show that (contrary to a commonly agreed doctrine in the literature) it is particularised properties which Bolzano thought to be the entities standing under the predicate-ideas of propositions. With this idea, a rather uncommon semantic arises : a proposition of the form $[A-$ has - (the property) $b$ ] will be true iff one of the particularised properties standing under the predicate-idea $[\mathrm{b}]$ inheres in the subject of the proposition, i.e. in the entity denoted by the subject-idea $[\mathrm{A}]$.

L’un des grands mérites de Bolzano est d'avoir légué à la postérité philosophique une théorie minutieuse et raffinée des propositions." Au sein de cette théorie, les propriétés (qualités) jouent un rôle important. Bolzano s'est efforcé d'établir ce qu'on pourrait appeler la «thèse $S$ » « $S$ » pour «structure »), c'està-dire la thèse selon laquelle les propositions ont une structure déterminée en vertu de laquelle elles énoncent (aussagen) des propriétés des objets sur

* Certaines parties de cet article, en particulier les sections 4 et 5, reprennent des résultats de mon livre Substanz und Adhärenz (Schnieder, à paraître). Mes remerciements pour leurs commentaires aux participants de l'Atelier-conférence Bolzano organisé par Kevin Mulligan à Genève en octobre 2001 et où j'en ai présenté une version préliminaire.

PHILOSOPHIQUES 30/1 - Printemps 2003, p. 83-103 
lesquels elles portent. Pour saisir la singularité de la position bolzanienne — et elle est bien plus singulière qu'on ne l'assume généralement - un examen approfondi de sa conception des propriétés est nécessaire. Je me propose de la présenter de manière détaillée.

Mon article s'articule de la manière suivante : après quelques brèves remarques préliminaires (section 1 ) je présente la thèse $S$ (section 2). J'examine ensuite deux particularités de la conception bolzanienne du concept d'énoncer (Aussagen) ou d'attribuer (Beilegen) qui distinguent sa position de certaines conceptions reçues (section 3). Afin d'expliquer la seconde particularité de la position bolzanienne - qui est aussi la plus importante — je m'intéresse ensuite à la notion bolzanienne d'adhérence (Adhärenz), c'est-à-dire à la notion de qualité particularisée concrète (section 4). Je démontre finalement que Bolzano attribut un rôle sémantique aux adhérences : les adhérences tombent sous les représentations-prédicats des propositions (qui traitent d'objets réels) — on trouve ainsi chez Bolzano l'esquisse d'une sémantique non standard que la littérature philosophique n'a pas, jusqu'à maintenant, pris en compte (section 5).

\section{Explications préliminaires : propositions et représentations}

Je prends pour acquis que le lecteur est familier avec les principes de base de la conception bolzanienne des propositions. Évoquons toutefois, afin d'éviter les désaccords tacites, quatre vecteurs essentiels de la position bolzanienne :

(i) Les propositions sont la signification (possible) ou le sens des assertions, ou le contenu (possible) des actes de jugements et des convictions. Je dis aussi d'une proposition qui est la signification d'une assertion donnée qu'elle est exprimée par cette dernière.

(ii) Les propositions sont, soit vraies, soit fausses, et elles le sont dans un sens absolu. Les propositions sont, de plus, les porteurs de vérité primitifs, ce qui veut dire qu'on ne peut expliquer l'idée de vérité ou de fausseté d'autres entités qu'en ayant recours à l'idée de vérité ou de fausseté des propositions.

(iii) Les propositions sont des entités non-réelles. Elles ne sont pas des entités possibles au sens où elles pourraient accéder à la réalité. Elles restent donc, en principe, extérieures à l'ordre causal et n'occupent aucune place dans la structure spatio-temporelle.

(iv) Les propositions sont des entités complexes dont les parties non-propositionelles sont appelées «représentations en soi » (ou encore : représentations objectives).

Bolzano fait usage de concepts méréologiques en rapport avec les propositions. Tout comme les énoncés sont composés de mots, les représentations sont composées de parties. Ces dernières peuvent certes aussi être des pro- 
positions, mais les propositions ont dans tous les cas des parties qui ne sont pas elles-mêmes des propositions, mais des représentations en soi :

(Rep) X est une représentation en soi $\leftrightarrow_{\text {Df }}$

$(\exists p)$ ( $p$ est une proposition $\& x<p \& \neg x$ est une proposition $)^{1}$

Par souci de concision, j'appellerai parfois les représentations en soi « concepts ». Je déroge à cet égard de la terminologie bolzanienne.

J'utilise les crochets carrés comme désignation pour les significations : une expression entre crochets carrés désigne la signification ou le sens de l'expression (exprimée dans un contexte donné) qui y est contenue. Par conséquent, [l'inventeur du moteur diesel] désigne un concept et [Cet inventeur était Russe] une proposition.

\section{La thèse $S$}

2.1 Formulation de la thèse $S$

Dans le second volume de la Wissenschaftslehre, Bolzano formule une thèse qui porte sur la structure générale de la proposition en soi (ou proposition). J'en examinerai différents aspects dans ce qui suit, mais voici d'abord, ce qu'en dit Bolzano :

On trouve dans toutes les propositions le concept d'avoir, ou plus précisément encore, le concept que désigne le mot a (hat). En plus de cette composante, toute proposition en présente encore deux autres que ce $a$ lie ensemble de la manière qu'indique l'expression : $A a b$. La composante qui est indiquée par $A$ se présente comme si elle devait représenter l'objet sur lequel porte la proposition, et $b$ comme la propriété que la proposition attribut à cet objet. Pour cette raison, je me permets de nommer cette partie $A$, quelle qu'en soit la teneur, la base ou représentation-sujet, $b$ par contre la partie assertive ou représentationprédicat $^{2}$.

Formulons maintenant la thèse $S$ :

(TS) Pour toute proposition $p$ :

il y a exactement une décomposition complète de $p$ en trois représentations $V_{1}-V_{3}$, tel que :

(i) $V_{1}$ est la représentation-sujet dont la fonction consiste à représenter l'objet sur lequel porte la proposition $p$ (dans ce qui suit, son sujet, par contraste avec sa représentation-sujet) \&

(ii) $V_{2}$ est la représentation-copule [a] \&

(iii) $V_{3}$ est la représentation-prédicat dont la fonction est de représenter une propriété que la proposition $p$ attribue à son sujet.

1. «<» désigne la relation de partie.

2. Bolzano, 1837, $\mathbb{1 2 7}$, vol. 2, p. 9. 
Explications : 1 . il y a exactement une décomposition de l'espèce en question - sinon la même proposition pourrait porter sur différentes choses et affirmer d'elles des choses différentes; elle pourrait de plus être vraie d'après une décomposition et fausse d'après une autre. 2. Une décomposition complète en $V_{1}-V_{3}$ ne signifie pas une décomposition en des parties qui n'ont plus elles-mêmes des parties (une telle décomposition livre en règle générale plus que seulement trois représentations). Une décomposition complète signifie bien plutôt une décomposition telle qu'il n'y pas de parties qui "soient de reste ", c'est-à-dire telle que $V_{1^{-}}$ $V_{3}$ composent précisément la proposition qui est analysée d'une manière déterminée. 3. Une représentation-sujet représente un objet : cela veut dire que cet objet tombe sous elle. 4. La représentation-sujet remplit une fonction, à savoir celle qui consiste à dénoter un objet. Toutefois certaines représentations-sujets échouent : elles sont vides. La condition (i) est donc plus qu'une simple variante stylistique de l'idée que «la représentation-sujet dénote l'objet sur lequel porte la proposition ». La même chose vaut à propos de la condition (iii).

En ce qui concerne notre investigation, la condition (iii) de (TS) est la plus importante et je l'examinerai donc en profondeur. Au préalable, dans le reste de cette section, j'avance quelques remarques préliminaires sur la signification de (TS) (et, en particulier, sur la manière dont (TS) ne doit pas être comprise).

\subsection{Le statut logique de la thèse $S$ et l'idée de paraphrase}

Bolzano ne dispose d'aucun argument conceptuel en faveur de la thèse $S$ et il s'efforce par conséquent de l'asseoir de manière inductive :

Afin de convaincre le lecteur de la rectitude de cette affirmation [à savoir (TS)], je ne connais de moyen plus exhaustif que de lui demander de chercher luimême à savoir, pour toute proposition qui se présente à lui, si elle peut être décomposée dans lesdites parties. Je me propose moi-même d'indiquer pour un nombre considérable de propositions, ou plus encore pour des genres entiers de propositions, les parties dont elles sont d'après-moi constituées $[\ldots]^{3}$.

Une bonne partie de la première moitié du second volume de la Wissenschaftslehre fait effectivement état de la tentative annoncée par Bolzano. Bolzano montre en quoi les propositions exprimées par une série d'énoncés de formes diverses ont en fait bel et bien toutes la même forme.

Bolzano parvient à ses fins en présentant une reformulation des énoncés initiaux. Il n'y a pas de consensus général sur le statut de cette reformulation au sein des études bolzaniennes. Textor s'est récemment attaqué à l'interprétation classique, à savoir que les reformulations bolzaniennes (que

3. Ibid., $\$ 127$, vol. 2 , p. 9. 
Textor appelle "paraphrases ») doivent être comprises comme préservant le sens. ${ }^{4}$ La position de Textor se rive aux deux thèses essentielles suivantes :

$\left(\mathrm{T}_{1}\right)$ Deux énoncés $s_{1}$ et $s_{2}$ expriment la même proposition (bolzanienne) seulement si elles sont congruentes (cela veut dire, grosso modo, qu'elles présentent la même structure grammaticale $)^{5}$.

$\left(\mathrm{T}_{2}\right)$ Les paraphrases proposées par Bolzano ne visent pas à conserver le sens, mais ont bien plutôt pour but la seule équipollence (c'est-à-dire l'équivéracité nécessaire avec l'énoncé paraphrasé) ${ }^{6}$.

Cependant, il me semble que l'attaque de Textor n'atteint pas sa cible, à savoir l'interprétation classique, et ce que dit Bolzano lui-même à ce sujet peut être mis à contribution pour le démontrer. Il y a deux arguments simples et probants contre $\left(\mathrm{T}_{2}\right)$ :

Bolzano ne parle pas de paraphrase pour caractériser son entreprise. Il a bien plutôt un concept spécial à sa disposition. Là où Textor dit que Bolzano paraphrase des énoncés (ou en offre une paraphrase), Bolzano dit qu'il tente de les interpréter (auslegen) (ou d'en offrir une interprétation (Auslegung)) ${ }^{7}$. Ainsi, jetant au paragraphe $\$ 169$ un regard rétrospectif sur les reformulations de propositions de formes diverses auxquelles il est déjà parvenu dans les pages précédentes, il écrit qu'il :

[...] n'a pas négligé d'introduire immédiatement les expressions linguistiques dans lesquelles les différentes espèces de propositions énumérées précédemment apparaissent habituellement; ce par quoi maintes formules qui auraient autrement nécessité une interprétation propre (eigene Auslegung), ont donc trouvé leur élucidation (Erläuterung) [... $]^{8}$.

Dans ses paraphrases, Bolzano vise donc à interpréter des énoncés linguistiques dont la structure grammaticale masque la structure logique. Et Bolzano ne laisse aucun doute sur ce en quoi consiste une telle interprétation : "Finalement, on nomme une proposition dans laquelle est énoncé que le sens de certains signes est celui-ci ou celui-là une interprétation (Auslegung) de ces derniers $»^{9}$.

On notera donc, pour anticiper de possibles scrupules, que ce ne sont pas les paraphrases qu'il entreprend qui constituent les interprétations dans son sens. Car une paraphrase n'énonce pas ce qu'est le sens de l'énoncé ainsi reformulé, mais — si la paraphrase est réussie — elle $a$ bien plutôt ce sens, elle le montre (et elle le fait d'une manière plus distincte que l'énoncé paraphrasé,

4. Par exemple, Casari (1992, p. 76) défend cette conception classique.

5. Textor, 1997, p. 190

6. Ibid., p. 198.

7. On trouve une discussion générale du concept bolzanien d'interprétation (située dans un contexte historique) dans Schmit, 1995.

8. Bolzano, 1837, $\mathbb{1 6 9}$, vol. 2, p. 211.

9. Ibid., $\$ 285$, vol. 3, p. 68 . 
car la structure de l'énoncé reformulé reflète, par contraste avec celle de l'énoncé initial, la structure de la proposition). Mais retournons à l'argument. La teneur du passage cité est simple et univoque : afin d'appuyer (TS) Bolzano s'efforce de trouver l'interprétation d'énoncés de formes diverses, et donc de donner leur sens. Par conséquent, Bolzano contredit directement $\left(\mathrm{T}_{2}\right)$.

Cependant, même s'il ne le faisait pas, il y aurait une autre bonne raison de douter de $\left(\mathrm{T}_{2}\right)$. Car, une chose est claire : les paraphrases de Bolzano constituent la pitance de son argument inductif pour (TS). Toutefois, il s'avère que sous l'interprétation de Textor, il ne peut en être ainsi. (TS) est une thèse concernant la composition de toute proposition. Si les paraphrases n'étaient pas effectivement conçues pour exprimer la même proposition que celle exprimée par l'énoncé paraphrasé, Bolzano chercherait ici un argument inductif des plus curieux : Si, lorsque la proposition exprimée par un énoncé donné n'est pas claire, Bolzano faisait appel à une autre proposition - équipollente et de la forme souhaitée - alors il trouverait bel et bien des instances positives de (TS), mais cela seulement au mépris des cas problématiques. Or, en procédant de la sorte, on ne gagne visiblement pas un argument inductif pour (TS) d'une manière analogue on pourrait argumenter que tous les nombres naturels sont pairs : en a-t-on un qui semble impair, alors on n'a qu'à le mette à l'écart et à considérer celui qui le suit; on trouvera ainsi naturellement un nombre infini d'instance positive de la thèse.

À la lumière de ces deux arguments, $\left(\mathrm{T}_{2}\right)$ et $\left(\mathrm{T}_{1}\right)$ se font irrécupérables. Car plusieurs des paraphrases citées par Bolzano ne sont précisément pas congruentes avec les énoncés initiaux ( «Il y a une licorne » devient «La représentation [licorne] est objectuelle») bien qu'elles soient supposées, comme on l'a mentionné, exprimer la même proposition. La congruence n'est donc pas nécessaire aux yeux de Bolzano.

Nonobstant le fait que je mène ici campagne contre les thèses de Textor, je ne veux surtout pas laisser entendre qu'elles seraient absolument non fondées. En tout cas, il vaut la peine de jeter un coup d'œil sur les passages sur lesquels Textor s'appuie. La section $\$ 137$ de la Wissenschaftslehre traite des énoncés de la langue courante de la forme «Il y a $\varphi$ » qui, comme nous l'avons mentionné, sont à comprendre d'après Bolzano comme des affirmations sur l'objectualité d'une représentation («La représentation $[\varphi]-\mathrm{a}-$ l'objectualité »). Bolzano termine le paragraphe avec une remarque circonspecte :

Si, malgré tout, quelqu'un devait trouver improbable que les interprétations des énoncés tentées ici indiquent vraiment la même pensée que celle que nous lions avec eux - car ces derniers sont composés de mots bien différents de ceux qu'on retrouve dans leur interprétation; eh bien, je ne veux pas me disputer là-dessus, mais je demande seulement que cette personne me concède que le sens que l'on lie avec ces expressions et le sens que j'en ai donné dans mon interprétation sont équivalents en ce qui concerne la signification, qu'à chaque fois que le premier énoncé est vrai, l'autre l'est aussi. Or, on pourra difficilement en disconvenir ${ }^{10}$.

10. Ibid., $\mathbb{S} 137$, vol. 2, p. 53s. 
Ici, donc, d'après la lecture de Textor, Bolzano concèderait lui-même que ses paraphrases ne conservent possiblement pas le sens et il se contenterait de moins (c'est-à-dire de l'équivalence ou de l'équipollence) ${ }^{11}$.

Il me semble néanmoins qu'on rate de la sorte l'intention de Bolzano. Ce qu'il concède, en fait, c'est que cette tentative d'interprétation pourrait être vouée à l'échec (ce qu'elle est effectivement si elle reflète de façon erronée le sens de l'énoncé interprété, c'est-à-dire si elle ne spécifie pas la proposition exprimée $)^{12}$. Et s'il en était ainsi, on devrait considérer deux problèmes additionnels :

(i) Que signifie l'échec de cette tentative particulière pour (TS) ? Certes, elle ne serait pas réfutée, mais elle serait tout de même remise en cause pour des énoncés de la forme en question ( «Il y a $\varphi$ »). Et il resterait à entreprendre, pour tâcher de la maintenir, une autre tentative d'interprétation, celle-là réussie, qui assignerait à l'énoncé la forme générale.

(ii) Mais il est important de considérer que, bien entendu, Bolzano n'a pas rédigé des centaines de pages dans le seul but d'étayer la thèse $\mathrm{S}$. Les interprétations qu'il offre de différentes espèces de propositions sont intéressantes pour les philosophes et logiciens indépendamment de cette thèse, et ce en particulier parce qu'elles nous permettent de parvenir à une plus grande clarté sur les règles d'inférence qui concernent ces espèces de propositions. Pour cette fin précise, l'identification d'un énoncé équipollent suffit (puisqu'il s'agit d'un énoncé logiquement équivalent). Si Bolzano ne veut pas ici se disputer sur la question de l'identité du sens de sa paraphrase, mais se dit content de concéder son équivalence, ce n'est donc pas parce qu'il considère que l'interprétation qu'il en donne est correcte. Elle peut néanmoins être utile, même si elle manque sa cible comme interprétation, ce avec quoi Bolzano peut se dire content dans le passage concerné.

\subsection{La lacune principale de la conception bolzanienne}

de la proposition et ses suites pour la thèse $S$

Mais comment, se demandera instinctivement la lectrice avertie, peut-on effectivement déterminer si une interprétation est réussie? Comment détermine-t-on quel est le sens d'un énoncé donné et si deux énoncés expriment la même proposition? Malheureusement, et c'est là un fait que Textor répète à satiété, Bolzano n'a pas une conception très claire de cela. Il aurait besoin de deux critères :

(C CE) Critère pour le contenu d'un énoncé (Étant donné un énoncé s, comment détermine-t-on quelle proposition $s$ exprime?)

11. Morscher comprend ce passage de la même manière : «Bolzano exige normalement des interprétations non seulement l'équivalence logique, mais l'identité du sens [...] Il arrive toutefois que Bolzano se satisfasse de l'équivalence logique », bien qu'il lui attribue une moindre importance, comme son «normalement» le montre; Cf. Morscher, 1973, p. 154 note.

12. Casari (1992, p. 77) semble comprendre ce passage de la même manière. 


\section{Philosophiques / Printemps 2003}

(C CM) Critère pour le contenu d'un mot (Étant donné un mot ou une locution $w$, comment détermine-t-on quel concept $w$ exprime ?)

Dans la mesure où la reformulation du sens exprimé par un certain assemblage de mots est elle-même donnée de manière linguistique, les critères spécifiés peuvent être reformulés de la manière suivante :

$\left(\mathrm{C}=\mathrm{CE}\right.$ ) Critère pour le contenu d'un énoncé (Étant donné deux énoncés $s_{1}$ et $s_{2}$, comment détermine-t-on si $s_{1}$ et $s_{2}$ expriment la même proposition ?)

$(\mathrm{C}=\mathrm{CM})$ Critère pour le contenu d'un mot (Étant donné deux mots ou locutions $w_{1}$ et $w_{2}$, comment détermine-t-on si $w_{1}$ et $w_{2}$ exprime le même concept ?)

On pourrait vouloir comprendre des critères de cette espèce comme des critères d'identités pour propositions ou concepts, mais prenons ici nos précautions. Car, Bolzano peut certainement offrir des conditions d'identité (et il arrive souvent qu'on parle de ces dernières lorsque se sont en fait des critères d'identité qui sont exigés) comparativement claires pour les propositions et les concepts :

(=P) Pour toute proposition $p_{1}, p_{2}$ :

$p_{1}=p_{2} \leftrightarrow p_{1}$ est composée de la même manière et des mêmes parties que $p_{2}$

(=B) Pour tout concept $b_{1}, b_{2}$ :

$b_{1}=b_{2} \leftrightarrow$

(i) $\quad\left(b_{1}\right.$ et $b_{2}$ sont complexes $\& b_{1}$ est composé de la même manière et des mêmes parties que $\left.b_{2}\right) \vee$

(ii) ( $b_{1}$ et $b_{2}$ sont simples \& les mêmes objets tombent nécessairement sous $b_{1}$ et $b_{2}$ )

Mais ce dont on a besoin pour pouvoir juger de la thèse $S$ est d'un critère qui fait le lien entre les expressions linguistiques et les entités propositionnelles, car c'est par les entités linguistiques que s'effectue l'accès aux entités propositionnelles ${ }^{13}$.

Cependant, Bolzano ne fournit ici aucun critère suffisant. Cela devient particulièrement manifeste lorsqu'il argumente en faveur de ses tentatives de définitions (ou analyses) des concepts. Quand une conditionnelle de la forme « $x$ est un $\mathrm{F} \rightarrow x$ est un $\mathrm{G}$ » livre-t-elle par surcroît une analyse du concept $[\mathrm{F}]$ ?

13. Je me limite ici aux affirmations concernant des entités linguistiques et leur sens, c'est-à-dire les concepts et propositions exprimés; on peut toutefois parler de manière analogue des états et processus mentaux. Bolzano n'a pas de meilleurs critères en réserve en ce qui concerne le niveau mental. 
Le passage suivant (qui, au demeurant, se rapporte à la tentative bolzanienne d'analyse du concept de vérité) pourrait servir de réponse à Bolzano :

On ne pourra prendre une telle conditionnelle comme définition que si elle se laisse inverser; si, de plus, aucun des concepts qui apparaissent ici ne contient de quelque manière que ce soit le concept [qu'on cherche à définir]; et si, finalement, il nous apparaît, lorsqu'on y réfléchi longuement, de plus en plus distinctement que nous ne pensons véritablement rien d'autre par le concept [qu'on cherche à définir] que celui qui est donné ici ${ }^{14}$.

Le résultat de cette "longue réflexion" est l'ultime critère que nous offre Bolzano. Cela ressemble toutefois un peu trop à de l'auto-suggestion méditative et paraît trop arbitraire pour être considéré sérieusement comme un critère. Ceci dit, il ne faut pas mal comprendre l'exigence d'un critère présentée ici; il ne s'agit pas de trouver un critère précis qui exclut tout vague. Le vague est peut-être inévitable et, pris isolément, il ne constitue pas une objection contre la théorie des propositions et des concepts. Nous avons cependant besoin d'un critère qui engendre, au moins dans plusieurs cas, des résultats clairs. Mais ce que nous offre Bolzano est, encore là, trop faible.

L'absence d'un critère fait en sorte que la thèse $S$ ne peut être testée de manière systématique. En ce qui a trait aux interprétations qu'il lui apporte comme support inductif, Bolzano est en fin de compte forcé de s'en remettre à la sympathie du lecteur (car même s'il n'y a pas d'argument clair contre elle, il pourrait certes y en avoir : si deux énoncés divergent, par exemple, à l'égard de leur valeur de vérité ou même seulement à l'égard de leur intension, ils n'expriment certainement pas la même proposition.) Un critère clair serait donc souhaitable, et on peut douter qu'on puisse en trouver un qui prouverait correctes toutes les interprétations de Bolzano.

\section{3. Énoncer une propriété}

Si la thèse $S$ est correcte, il devrait y avoir pour tout énoncé sensé qui n'a pas la forme «A a $b$ » un autre énoncé qui exprime cette proposition et qui a cette forme ( « devrait» et non pas «doit»; la formulation la plus forte n'est valable que sous la supposition additionnelle que le pouvoir expressif de notre langue est assez fort - cette supposition est raisonnable, mais je n'argumenterai pas en sa faveur ici). Ceci est moins manifeste pour les énoncés complexes ou pour ceux qui contiennent des quantificateurs que pour les énoncés atomiques ou ceux qui n'en contiennent pas. Comment Bolzano tente-t-il de rendre sa thèse plausible à l'égard des énoncés atomiques et de ceux ne contenant pas de quantificateurs, et dans quelle mesure y parvient-il ? 
C’est au paragraphe $\$ 127$ que Bolzano argument en faveur de la thèse $\mathrm{S}$ en ce qui concerne les énoncés atomiques. Il y suggère que :

Il est encore plus évident que les propositions de la forme : $A$ est $B$, n'ont aucune autre sens que celui que l'expression : $A$ a $b$, indique, pour autant que $b$ représente l'abstractum qui correspond au concreto $B^{15}$.

Bolzano explique ce qu'il entend par un concretum et un abstractum dans le premier volume de la Wissenschaftslehre ${ }^{16}$. Un abstractum est tout simplement une représentation de propriété; a-t-on une représentation de propriété $[b]$, le concretum qui lui correspond est la représentation [quelque chose, qui a $b$ ]. Par exemple, la représentation [rouge] est exprimée de manière plus appropriée par [quelque chose, qui a la rougeur] et est donc le concretum qui correspond à l'abstractum [rougeur]. De la même manière, [un cheval] est le concretum de [chevalinité], car il serait mieux exprimé par [quelque chose, qui à la chevalinité ${ }^{17}$.

Seulement, comme l'exemple précédent le montre, il n'y a pas un mot approprié pour chaque abstractum - un trait qui n'est pas exclusif à la langue française ou allemande et dont Locke se sert pour critiquer l'idée selon laquelle tous ces abstracta subsistent réellement ${ }^{18}$. Mais, on peut ici se servir d'une construction : "La propriété d'être F ", qui ne brille toutefois pas par son élégance. Dans les formulations schématiques, j'utilisera pour être bref «F-ité » comme substitut pour le terme abstrait qui correspond au terme général « $\mathrm{F} »$. Bolzano souscrit donc, dans la citation précédente, au schéma général :

\section{(BP) « $x$ est $\mathrm{F} »$ exprime la même proposition que « $x$ a la F-ité »}

Suivant la thèse $S$, toute proposition contient une représentation-prédicat ou partie assertive qui est supposée représenter ce qui est énoncé de l'objet de la proposition. Ce qui est énoncé, selon la terminologie bolzanienne, sont des propriétés, et ce qui énonce ces propriétés sont les propositions. Remarquons en passant que la terminologie bolzanienne pourra paraître étrange au profane. Normalement, on dit que des personnes énoncent quelque chose, et on spécifie ce que ces personnes énoncent par une nominalisation de l'énoncé : «le témoin a dit que l'accusé était l'auteur du hold-up». Apparemment, dans

15. Ibid., $\mathbb{S} 127$, vol. 2, p. 10 .

16. Ibid., $\mathbb{S} 60$, vol. $1, \mathrm{p} 259 \mathrm{~s}$.

17. On voit que l'usage que fait Bolzano de «concret » et «abstrait " n'a rien à voir avec la distinction usuelle entre objets concrets et abstraits. Non seulement utilise-t-il ces termes pour une distinction qui concerne les représentations et non pas les objets, mais plusieurs représentations d'objets que les philosophes contemporains diraient «abstraits" sont, au sens de Bolzano, concrets : [quelque chose qui n'a pas de position dans la structure temporelle], [Nombre], [Ensemble], etc. Par contre les représentations d'adhérences (voir ci-dessous) sont, d'après Bolzano, abstraites malgré que leurs objets sont aujourd'hui généralement désignés comme concrets.

18. Locke, 1975, Livre III, Chapitre VIII, p. 474s. 
ce sens, ce qui est énoncé ici ne sont pas des propriétés, mais des propositions. Certes, il existe plusieurs tournures du langage ordinaire qui correspondent à l'usage bolzanien. On attribut à Johanne la droiture d'esprit, on impute souvent l'orgueil à Napoléon. On ne peut donc pas simplement tourner le jargon bolzanien à la dérision puisqu'il s'appuie sur un usage qui nous est quotidien.

Les philosophes auront quant à eux peu ou pas de problèmes avec la terminologie bolzanienne car il existe une tradition philosophique qui remonte jusqu'à Aristote et pour laquelle les attributs et les propriétés doivent être conçus comme ce qui est énoncé ou prédiqué. Mais on doit éviter d'assimiler trop rapidement la position de Bolzano à celles qui sont déjà connues. La conception bolzanienne de ce en quoi consiste l'énoncer d'une propriété se distingue d'autres positions largement répandues au moins en sous deux aspects.

Premièrement, Bolzano exige des représentations-prédicats qu'elles soient des représentations de propriétés, sans toutefois imposer quelque contrainte supplémentaire que ce soit sur leur forme ${ }^{19}$. Cela signifie, en particulier, que les représentations qui sont exprimées par des marques distinctives sont acceptées à titre de représentations-prédicats. D'après Bolzano, les deux énoncés suivants exprimeraient des propositions structurellement transparentes, (le sujet grammatical exprime la représentation-sujet, le complément grammatical la représentation-prédicat) :

(1) Orson - a - mon vice préféré.

(2) Orson - a - une propension exagérée à faire des câlins.

Bien entendu, (1) et (2) n'expriment pas la même proposition; car si mes prédilections étaient différentes, (1) pourrait être faux et (2) vrai. Mais, et cela est bien une particularité de la position bolzanienne, si mon vice préféré est de facto la tendance exagérée à faire des câlins, alors les deux propositions exprimées ici d'une manière structurellement transparente disent la même chose d'Orson (c'est-à-dire, précisément : la même propriété) ${ }^{20}$. Ainsi, on trouve chez Bolzano un principe (PE) concernant le concept d'énoncer :

(PE) Pour toute proposition $p, q$ : si la même propriété est attribuée au même sujet représenté de la même manière dans $p$ comme dans $q$, alors :

$p=q$.

19. À d'autres endroits, Bolzano semble ne pas en exiger autant de la représentationprédicat (voir les remarques de Siebel (1996, p. 33s) sur l'idée d'une conception plus libérale de la proposition en soi chez Bolzano). Néanmoins, les constructions qui n'ont pas une représentation de propriété dans la position du prédicat ne peuvent pas, selon Bolzano, être vraies : « Si en plus de cela, la proposition en question doit être vraie [...] les deux parties $A$ et $b$ doivent être des représentations et, en fait, des représentations objectuelles, et en particulier $b$ doit représenter une propriété.» (Cf. Bolzano, 1837, \$127, vol. 2, p. 16.) Ceci laisse toutefois ouverte la question de la forme de la représentation de propriété.

20. Je ne connais aucun passage où Bolzano souligne ce trait de sa théorie explicitement. Mais il découle simplement et directement de la conception bolzanienne : on se rappellera par exemple la caractérisation de la représentation-prédicat citée au début de l'article : «comme si 
Deuxièmement, supposons que les deux énoncés suivants expriment des propositions vraies :

(3) Johanne a le courage.

(4) Socrate a le courage.

Cela semble impliquer qu'il y a une propriété $b$ dont on peut dire : (i) $b$ tombe sous la représentation-prédicat de la proposition exprimée par (3); (ii) $b$ tombe sous la représentation-prédicat de la proposition exprimée par (4) et (iii) $b$ est une propriété tant de Socrate que de Johanne. La propriété en question est le soi-disant courage, et puisque qu'elle peut revenir à deux personnes différentes en même temps, il s'agit d'un universel. Dans les deux prochaines sections je démontrerai que Bolzano n'est pas d'accord avec cette idée traditionnelle. Il a une autre conception des propriétés, une conception particulièrement fertile dans les contextes sémantiques.

\section{Les adhérences}

4.1 Les adhérences sont des propriétés réelles

Bolzano défend une ontologie bicatégoriale du réel; on la trouve résumée de façon significative dans l'Athanasia:

Tout ce qui est, c'est-à-dire tout ce qui subsiste en réalité, [...] appartient à une des deux espèces suivantes : soit cela est et subsiste dans quelque chose d'autre, comme propriété de cette chose, soit cela n'est pas une simple propriété de quelque chose d'autre mais subsiste, comme on a l'habitude de dire, en soi [...]. Les philosophes ont aussi l'habitude d'appeler les réalités de la première espèce des adhérences, et celles qui appartiennent à la seconde des substances ${ }^{21}$.

Nous réserverons un rôle de figurant aux substances dans ce qui suit, et une présentation rapide devrait suffire ici : les substances sont des choses du monde de la vie de tous les jours, les corps animés et inanimés (les ascarides, les autruches, les tasses à café, le mont Everest et la planète Terre), les personnes, et d'après la métaphysique bolzanienne, les âmes (des substances atomiques dotées d'une faculté de représentation qui occupent des points spatiaux individuels), les portions et les masses de matière (le café dans ma Tasse ou le Lac-St-Jean), et enfin aussi des collections de substances, comme la Voie Lactée ou, en fin de compte, l'univers englobant toutes les substances créées.

elle devait représenter [...] la propriété que la proposition attribut à cet objet. Pour cette raison, je me permets de nommer cette partie [...] $b$ la partie assertive ou représentation-prédicat " (Bolzano, 1837, II, $\mathbb{S} 127$, p. 9). Quelle propriété est énoncée du sujet (ou lui est attribuée) dans une proposition dépend de ce qui tombe sous la représentation-prédicat. Deux représentationsprédicats différentes conditionnent dans tous les cas deux propositions différentes, mais elles peuvent parfaitement avoir la même extension et autorisent donc l'énoncer de la même propriété.

21. Bolzano, 1838, p. 21 
Tournons-nous maintenant vers les adhérences; la citation précédente nous fournit déjà une définition du concept [adhérence] :

(Df. Adh) $x$ est une adhérence $\leftrightarrow_{\text {Df. }} x$ est une propriété $\& x$ est réel.

Que veut dire ici «réel»? Le concept de réalité est un concept primitif et non-analysable de l'ontologie bolzanienne. Parmi tout ce qu'il y a, tous les objets, choses et autres entités, il y en a qui n'ont pas la réalité, comme les propositions, les concepts et les nombres. Les choses réelles entretiennent, par contraste avec ces dernières, des relations causales (elles agissent ${ }^{22}$ les unes sur les autres, sont des causes et des effets) et elles se trouvent dans le temps et l'espace ${ }^{23}$.

Mais dans quelle mesure les propriétés sont-elles réelles, c'est-à-dire des causes et des effets? Bolzano peut appuyer sa conviction qu'elles le sont sur des données du langage familier. D'une part, on avouera qu'on parle parfois des événements comme causes et effets, et Bolzano comprend les événements comme une espèce de propriétés dynamiques (de telle sorte qu'un $\varphi$ 'er - un marcher, un exploser, etc. - est la propriété de $\varphi$ 'er). Le fait de ranger les événements au nombre des propriétés est peut-être inhabituel et discutable, mais on trouve des propriétés au sens tout à fait classique comme relata dans des énoncés causaux :

(1) L'étourderie de Clouseau lui fit envoyer l'inspecteur Dreyfus par le fond (c'est-à-dire : fut la cause de la chute de Dreyfus dans le lac).

(2) La défaut matériel de la machine causa l'incendie.

(3) Le courage de Johanne entraîna sa victoire (ou : la causa).

Bolzano prend au mot de telles tournures de phrases et tient pour cette raison (plusieurs) propriétés pour réelles. La conséquence directe de l'idée que les adhérences sont réelles est qu'elles se trouvent aussi dans le temps : le défaut matériel de la machine exista pendant un certain laps de temps et conduisit à l'anéantissement de sa propre existence et de celle de la machine dont il était la propriété. Mais Bolzano semble refuser aux adhérences une position spatiale, ce qui, à première vue, sera surprenant. Les événements comptent au nombre des adhérences, mais n'ont-ils pas lieu dans certains endroits? On s'exprime de cette manière : "Où la pièce fut-elle jouée ? " «On peut, encore aujourd'hui, fixer la position exacte du naufrage du Titanic.» « Notre rencontre eut lieu à proximité du musée. » Et ne pourrait-on pas dire que la pâleur de cet homme là-bas a un certain emplacement (il est si pâle

22. Les termes allemands pour "agir » (wirken) et « réalité » (Wirklichkeit) ont une racine étymologique commune. Note de l'éditrice.

23. Avec quelques exceptions : (i) seules les entités créées se trouvent dans l'espace; une entité divine, par contre, non; (ii) dans certains passages, Bolzano place Dieu à l'extérieur du temps; (iii) il arrive que Bolzano écrive que seules les substances se trouvent dans l'espace. 


\section{Philosophiques / Printemps 2003}

autour du nez!) et assurer de la sorte un emplacement spatial à certaines adhérences non dynamiques?

Même si la définition bolzanienne de l'espace ne consent un emplacement qu'aux substances, il lui est tout de même possible de réagir à nos exemples en concédant que les adhérences ont un emplacement dans un sens dérivatif. Nous disons qu'elles se trouvent ici ou là parce que leur porteur s'y trouve. On trouve la pièce là où se trouvent les acteurs qui la jouent. Le naufrage du Titanic eu lieu là où le Titanic se trouvait à cet instant. Le lieu de notre rencontre dépend de notre emplacement. Une telle manœuvre est aussi à la disposition de Bolzano dans le cas de la pâleur de l'homme : dire qu'elle se trouve autour de son nez spécifie où sont les parties pâles de l'homme, et donc où se trouvent les parties de son corps dans (non pas au sens spatial mais au sens attributif) lesquelles est la pâleur. L'attribution d'un emplacement est donc encore là dérivatif.

Les adhérences sont des propriétés réelles. Mais quelles propriétés sont réelles, et lesquelles ne le sont pas? Bolzano soutient que

[...] nous pouvons et devons attribuer l'être (Dasein) [c'est-à-dire la réalité] à un attribut lorsque [...] l'objet dans lequel cet attribut se trouve, a lui-même l'existence ${ }^{24}$.

Toutes et seules les propriétés des objets réels sont elles-mêmes réelles, donc des adhérences.

\subsection{Les adhérences sont individuées par leur porteur}

Tandis qu'il arrive souvent qu'on conçoive les propriétés comme des objets universaux qui peuvent revenir à plusieurs objets, les adhérences bolzaniennes sont des entités individuelles ou, plus précisément, des entités individuées par leur porteur. Ainsi, Bolzano écrit par exemple au sujet des représentations subjectives (qui sont les adhérences d'une substance mentale; au demeurant il écrit quelque chose de comparable au sujet des jugements ${ }^{25}$ ) :

Il est tout aussi évident que la représentation que j'ai de cet arbre, et la représentation que mon compagnon a du même objet, ne doivent pas être considérées comme un seul et même objet, mais bien plutôt comme deux représentations, et ce certes nonobstant toutes les coïncidences qui peuvent se trouver entre elles ${ }^{26}$.

Même lorsque deux personnes pensent au même objet de la même manière, lorsque, par exemple, elles saisissent toutes deux une représentation qui a le contenu [l'inventeur de la pompe hydraulique], chacune d'elle a une représentation propre, numériquement distincte de celle de l'autre. Il est

24. Bolzano, 1837, $\mathbb{S} 80$, vol. 1, p. 387; Voir aussi, par exemple, Bolzano, 1838, p. 22, Bolzano/Exner 1935, p. 79, de même que Bolzano, 1837, $\$ 142$, vol. 2, p. 65.

25. Ibid., $\$ 292$, vol. 3, p. 112.

26. Ibid., $\mathbb{S} 273$, vol. 3, p. 12. 
difficile de voir pourquoi les adhérences psychiques devraient être distinguées, à cet égard, des autres adhérences. Et on trouve effectivement dans la correspondance avec Exner le passage suivant :

Ce rouge (numero idem) ne peut se trouver dans deux roses. Le rouge qui se trouve dans une deuxième rose peut nous apparaitre, si vous le voulez, identique, tout à fait identique (gleich), mais il ne peut être le même parce que ce n'est pas la même rose : deux roses nécessitent deux rouges $^{27}$.

Bolzano parle ici comme si cela allait de soi et ne semble pas penser qu'un argument soit nécessaire. Mais on peut facilement extraire un tel argument de la définition des adhérences comme propriétés réelles. Le rouge de cette rose, quelle que soit sa ressemblance avec celui d'une autre, et même s'il est d'un ton identique, se tient certainement dans des relations causales différentes. On peut voir le premier sans voir le second; on peut, en détruisant la première rose, détruire le premier sans toucher au second. Si on reconnaît aux propriétés le statut de relata causaux, on ne peut alors les concevoir comme des universaux, mais on doit bien plutôt les concevoir comme des objets individuels concrets. Autrement, on prend pour acquis des relations causales qui n'existent pas.

Mais quel est le degré d'exactitude avec lequel on peut définir la propriété d'être individuée par son porteur qu'on attribue ici aux adhérences? Les adhérences reviennent à des choses, sont dans elles, leurs sont inhérentes. Avec ce concept primitif, on peut, pour exprimer que les adhérences n'inhèrent qu'à un seul porteur, formuler l'énoncé suivant :

(TI) Pour toute adhérence a, $b$, et tout objet $x, y$ :

( $a$ inhère à $x \& b$ inhère à $y \& x \neq y) \rightarrow a \neq b^{28}$.

\section{La sémantique non-standard de Bolzano}

5.1 La thèse de l'équivocité et la sémantique standard

La littérature qui touche à la théorie sémantique de Bolzano fait rarement mention des adhérences. On pourrait vouloir imputer ce mépris à l'idée que leur rôle se trouve ailleurs. Mais les propriétés jouent certainement un rôle pour la conception bolzanienne de la proposition. Les propriétés sont énoncées dans des propositions, elles se tiennent sous les concepts-prédicats des propositions. Comment la théorie des adhérences pourraient-elles ne pas être partie constituante de la sémantique bolzanienne?

27. Bolzano/Exner 1935, p. 32s.

28. Levinson (1980, p. 114) de même que Lehrer et McGee (1992, p. 43) jettent le doute avec des exemples comme le suivant : Le rouge d'une pomme, semble-t-on vouloir dire, n'est pas différent de celui de sa pelure. Mais une pomme et sa pelure ne sont pas une seule et même chose, et cet exemple semble donc contredire (TI). Je discute de la manière dont on doit réagir à de tels exemples dans Substanz und Adhärenz, chapitre I.4 (à paraître). Pour les fins de la présente discussion, (TI), même s'il nécessite possiblement encore quelques modifications, devrait suffire. 
Tournons-nous vers les rares spécialistes de Bolzano qui ont tenu compte de sa conception des adhérences et qui ont cherché à répondre à cette question. Künne, Textor et Chisholm ont tous pris note de la notion bolzanienne d'adhérence. Cependant, ils défendent tous la thèse que Bolzano utilise l'expression "propriété » de manière équivoque ${ }^{29}$. Bolzano aurait d'après eux deux conceptions différence des propriétés : d'une part, il tiendrait les propriétés pour des entités universelles et abstraites, ce qu'on peut aussi appeler des attributs. D'autre part, Bolzano les tiendrait pour des propriétés individuées par leur porteur qui sont réelles dans la mesure où leur porteur l'est aussi, et donc pour des adhérences. On peut toutefois considérer les attributs comme des espèces dont les adhérences sont des instances et défendre le schéma suivant :

(AA) $x$ a (exemplifie) l'attribut $a$ ssi $\exists b$ ( $b$ est une instance de $a$ (c'est-à-dire une adhérence) $\& x$ a $b$ ( $b$ inhère à $x)$

On notera ici la distinction entre la relation d'instanciation (entre le courage de Johanne et l'attribut courage), celle d'exemplification (entre Johanne et l'attribut courage) et celle d'inhérence (entre le courage de Johanne et Johanne).

On pourrait maintenant défendre, en conjonction avec la thèse de l'équivocité, que ce sont des attributs qui tombent sous les représentations-prédicats et qui remplissent une fonction sémantique, tandis que les adhérences ont bien plutôt leur place dans l'ontologie de la réalité. En combinant ces deux thèses on obtiendrait donc la réponse suivante à la question du rôle des adhérences dans la sémantique bolzanienne :

(TE) Bolzano utilise "propriété » de manière équivoque; d'un côte comme terme désignant des attributs, de l'autre comme terme désignant les instances des attributs, c'est-à-dire des entités individuées par leur porteur.

(RS) Le rôle sémantique des propriétés (elles sont énoncées et tombent sous la représentation-prédicat des propositions) est remplit par les attributs et non par les adhérences.

D'après moi, il y a de bonnes raisons de douter de la thèse de l'équivocité $(\mathrm{TE})^{30}$. Quoi qu'il en soit, il y en a de meilleures encore de douter que la sémantique esquissée par (RS) constitue une interprétation correcte de Bolzano. Je vais me contenter ici de présenter cette dernière et de défendre l'idée que même si Bolzano accepte les attributs, ce ne sont pas ces derniers mais les adhérences qui remplissent le rôle de propriétés dans sa théorie sémantique. J'esquisserai ensuite la sémantique qui en résulte.

29. Chisholm 1986, 1989, Künne 1998, p. 239s et Textor 1996, p. 63s.

30. Voir là-dessus Schnieder, à paraître, Chapitre I.5. 


\subsection{Contre (RS) et donc contre l'attribution d'une sémantique standard à Bolzano}

En ce qui à trait à l'argumentation contre (RS), je prendrai le chemin le plus facile : je laisserai parler Bolzano. Au paragraphe $\$ 143$ de la Wissenschaftslehre, Bolzano traite des propositions qui attribuent à l'être pensant des épisodes psychiques (jugements, représentations subjectives, sensations, etc.); au cours de cette attribution :

[...] sont générées des propositions de la forme : A - a - la représentation B; $\mathrm{A}$ - a - (ou porte $(f a ̈ l l t))-$ le jugement $\mathrm{C}$; $\mathrm{A}-\mathrm{a}-$ la sensation $\mathrm{D}[\ldots]$, et on peut extraire (entnehmen) de ces expressions la manière dont je conçois la structure logique des ces propositions ${ }^{31}$.

Il n'est pas difficile de procéder à cette "extraction ", car toutes les propositions que présente ici Bolzano ont la forme canonique : " $A$ a $b$ ». Les propositions en question ont ici les représentations-prédicat suivantes : [la représentation (subjective) B], [le jugement C], [la sensation D]. Or, les représentations subjectives, les jugements et les sensations sont des adhérences et non pas des attributs. En l'occurrence, ce sont donc des adhérences et non pas des attributs qui tombent d'après Bolzano sous les représentations prédicats ${ }^{32}$.

Les adhérences psychiques ne font ici pour Bolzano nullement figure d'exception. Dans la Wissenschaftslehre, Bolzano discute la doctrine kantienne de la forme du jugement et donne deux exemples de propositions en soi, à savoir [la distance a une grandeur] et [L'omnipotence est une propriété que seul Dieu a], et défend que :

Dans les exemples en question, les représentations-sujets et - prédicats sont reliées par le même concept de liaison, à savoir le concept d'avoir, à travers lequel la représentation d'une substance est connectée avec celle de son adhérence ${ }^{33}$.

D’après Bolzano, la copule "avoir » connecte des représentations de substances avec des représentations d'adhérences. La thèse qui veut que les adhérences ne remplissent aucune rôle dans la sémantique bolzanienne s'avère donc manifestement fausse ${ }^{34}$.

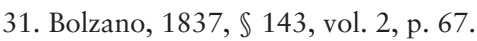

32. Bolzano réitère à plusieurs endroits cette conception des propositions qui portent sur des épisodes psychiques. Cf. par exemple Bolzano, 1838, p. 54s.; 1838, p. 290; 1837, $\mathbb{2} 271$, vol. 3, p. 8s. et ibid., $\mathbb{\$} 300$, vol. 3, p. 131.

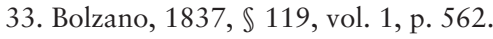

34. D'autres passages de Bolzano : Dans l'Athanasia (Bolzano, 1838, p. 34) il parle de manière indifférenciée de l'attribution de propriétés et de changements (et donc d'adhérences); À la section $\ 142$ de la Wissenschaftslehre (p. 65) Bolzano répète d'un trait que chaque propriété d'une chose réelle est elle-même réelle et que chaque propriété d'un objet peut lui être attribuée dans une proposition de la forme " $\mathrm{A}-\mathrm{a}-b$ ». Enfin, Bolzano défend la thèse que je lui attribue explicitement et la formule à nouveau de manière générale à la section $\mathbb{} 192$ du même ouvrage (p. 303). 
5.3 Une esquisse de la sémantique non standard de Bolzano

L'idée bolzanienne selon laquelle des adhérences tombent sous les représentations-prédicats constitue une ligne directrice intéressante, mais Bolzano n'a pas élaboré la théorie sémantique correspondante dans le détail. On peut toutefois brosser une esquisse de cette théorie sur la base de ses instructions.

Par souci de simplicité, je ne considère dans ce qui suit que des propositions dont le sujet est un objet réel (et c'est à elles que je réfère lorsque je parle de proposition). Les cas les plus simples présentent des propositions dans lesquelles une propriété est attribuée à une substance, et dont l'expression schématique est :

\section{(AS) (la substance) $x$ a $b$}

Sous la représentation $[b]$ tombent des adhérences. Celles qui tombent sous la représentation [courage] sont, par exemple, le courage de Johanne et le courage de Socrate, le courage de John Maynard, etc. La proposition a des conditions de vérité qui sont claires et qu'on stipulera préférablement sur base de la définition bolzanienne de la vérité :

(Vrai) la proposition $p$ est vraie $\leftrightarrow_{\text {Df. }}$

(i) la représentation-sujet $V_{1}$ de $p$ est objectuelle $\&$

(ii) $\forall x\left(x\right.$ tombe sous $V_{1} \rightarrow \exists y$ (y tombe sous la représentation-prédicat de $p \& x$ a $y))^{35}$.

Si une adhérence qui tombe sous la représentation-prédicat revient au sujet, alors la proposition est vraie. Ainsi, [Johanne a le courage] est vraie, car le courage de Johanne tombe lui aussi sous la représentation [courage]. [Cette poule mouillée-là a du courage] est fausse, car aucune des adhérences qui tombent sous la représentation [courage] ne revient à cette poule mouillée : il n'y a pas une telle chose que le courage de cette poule mouillée-là.

Mais qu'en est-il des affirmations qui concernent des propriétés? Il n'en est pas autrement lorsqu'il s'agit d'une affirmation sur une adhérence déterminée :

\section{(AA) (l'adhérence) $a$ a $b$}

[L'ironie de Morissey a du mordant] est vraie, car toutes les adhérences de mordant, et cela vaut donc pour le mordant de l'ironie de Morissey, tombent sous la représentation [mordant].

Un cas difficile pour terminer. Je n'ai certes pas démontré ici qu'il n'y a pas de place dans l'ontologie de Bolzano pour les attributs - et la question n'est donc ici qu'hypothétique - mais on peut se demander comment, s'il n'y avait pas, en plus des adhérences individuelles, l'attribut qui leur correspond

35. Voir Bolzano/Exner 1935, p. 90 ; Bolzano, 1837, $\mathbb{S} 131$, vol. 2, p. 26 et Bolzano Gesamtausgabe, II A, 12/2, p. 105. 
(c'est-à-dire, s'il n'y avait pas en plus du courage de Johanne, du courage de Socrate, etc. un attribut abstrait courage), on devrait transiger avec des expressions générales qui portent sur les propriétés comme "le courage est une vertu » ou "rouge est une couleur». Si les termes de propriétés dans les affirmations générales de cette espèce sont employés de la même manière que dans les propositions précédentes, ils expriment des représentations sous lesquelles tombent des adhérences. L'utilisation du singulier doit donc être comprise, dans ce cas comme dans les autres, comme une marotte de la langue. Cependant, il arrive qu'on emploie effectivement le singulier de manière similaire lorsqu'on a affaire à des substances. On dit que l'homme est mortel mais on ne parle pas d'un homme particulier mais de tous les hommes (c'est du moins la compréhension qu'à Bolzano de cette tournure de phrase) ${ }^{36}$. On dit la même chose, mais peut-être d'une manière un peu plus transparente avec l'énoncé «Tout homme est mortel».

On peut se servir de ce modèle pour les affirmations qui portent sur des propriétés. On peut ramener «le courage est une vertu » à la forme standard en deux étapes. On s'occupe d'abord du prédicat et on parvient alors à "Le courage a (la propriété) d'être une vertu » et on énonce ensuite explicitement le quantificateur universel qui se camoufle sous l'usage du singulier : "Tout courage a (la propriété) d'être une vertu ». D'une manière analogue on obtient à partir de «Rouge est une couleur » l'énoncé "Tout rouge a (la propriété) d'être une couleur ». Le premier énoncé exprime une proposition vraie, car tout objet qui tombe sous la représentation-sujet, c'est-à-dire tout courage, a une adhérence, à savoir, celle d'être une vertu. Et tout rouge a également une adhérence du type être une couleur.

Pour parler en termes généraux, la stratégie consiste ici à comprendre les affirmations de la forme :

(la propriété) $b$ a $c$

comme exprimant

Toute (adhérence) $b$ a $c$

Bien entendu, une telle position pose certaines difficultés et suscitera des questions, mais celles-ci dépassent le cadre de notre introduction au modèle bolzanien. Nous espérons toutefois avoir atteint notre objectif et avoir convaincu le lecteur qu'il est erroné d'assimiler la sémantique bolzanienne et son concept central d'énoncer une propriété avec le concept sémantique classique de l'attribut comme entité énoncée. Bolzano offre un modèle alternatif hautement intéressant qui mérite une investigation détaillée. Terminons en mentionnant que l'étude de Mertz (1996) montre de manière impressionnante ce que peut permettre une telle position : il y développe une 
sémantique formelle complète qui concordent dans ses grandes lignes avec les traits principaux de notre esquisse du modèle bolzanien ${ }^{37}$.

(Traduit de l'allemand par Sandra Lapointe)

\section{Bibliographie}

Bolzano, Bernard, Athanasia, Sulzbach, 1838. (Réimprimé : Frankfurt am Main, 1970)

Bolzano, Bernard, Bernard Bolzano - Gesamtausgabe, dir. E. Winter, J. Berg, F. Kambartel, J. Louzil, B. van Rootselaar, Stuttgart-Bad Cannstatt, Fromann Holzboog, 1969-...

Bolzano, Bernard, Wissenschaftslehre, Sulzbach, 1837 (Réimprimé : Leipzig 1929, 1981).

Bolzano, Bernard/Exner, Franz, Der Briefwechsel B. Bolzano's mit F. Exner, Prag, 1935.

Casari, Ettore, «An Interpretation of some Ontological and Semantical Notions in Bolzano's Logic ", Proceedings of the Meeting (1987) of the Florence Center for the History and Philosophy of Science : Bolzano's Wissenschaftslehre 1837-1987, Florence, 1992, p. 55-105.

Chisholm, Roderick, "On the Positive and Negative States of Things ", Grazer Philosophische Studien 25/26, 1986, p. 97-106.

Chisholm, Roderick, "Bolzano on the Simplicity of the Soul ", dans Traditionen und Perspektiven der analytischen Philosophie, Festschrift für Rudolf Haller, dir.

Gombocs, W./Rutte, H./Sauer, W., Vienne, 1989, p. 79-88.

Künne, Wolfgang, "Substanzen und Adhärenzen - Zur Ontologie in Bolzanos Athanasia ", Philosophiegeschichte und logische Analyse,1, 1998, p. 233-250.

Lehrer, Keith/McGee, Vann, "Particulars, Individual Qualities, and Universals ", dans Language, Truth and Ontology, dir. Mulligan, Kevin, London, Kluwer, 1992, p. 37-47.

Levinson, Jerrold, "The Particularisation of Attributes», The Australasian Journal of Philosophy, 58, 1980, p. 102-115.

Locke, John, An Essay Concerning Human Understanding, dir. von Nidditsch, H., Oxford, Clarendon Press, 1975.

Mertz, D. W., Moderate Realism and its Logic, New Haven/London, Yale University Press, 1996.

37. Si j'ai raison de dire qu'il n'y a pas chez Bolzano de catégorie d'attribut, alors ma position diffère de manière importante de celle de Mertz. Ce dernier assume, en plus des «property instances» (les propriétés individuées par leur porteur) il y a aussi les types de ces dernières, c'està-dire des attributs, comme entités à part. Cependant, et c'est là l'affinité essentielle avec Bolzano, la position du prédicat dans une affirmation est réservée aux désignations d'instances d'attributs. Dans la sémantique de Mertz, les désignations d'attributs ne peuvent apparaître que dans la position du sujet. 
Bolzano sur la structure des propositions et le rôle sémantique des propriétés · 103

Morscher, Edgar, Das logische An-Sich bei Bernard Bolzano, Salzburg, Anton Pustet, 1973.

Morscher, Edgar, «Hintertürln für Paradoxien in Bolzanos Logik », Philosophia Naturalis, 24, 1987, p. 414-422.

Schmit, Roger, «Über Bolzanos Begriff der Auslegung ", Grazer Philosophische Studien, 7, 1994, p. 1-29.

Schnieder, Benjamin, Substanz und Adhärenz - Bolzanos Ontologie des Wirklichen, Sankt Augustin, Academia Verlag, à paraître.

Textor, Mark , Bolzanos Propositionalismus, Berlin/New York, de Gruyter, 1996.

Textor, Mark, «Bolzano’s Sententialism », Grazer Philosophische Studien, 53, 1997, p. 181-202. 